Published in final edited form as:

Curr Opin HIV AIDS. 2016 May ; 11(3): 294-300. doi:10.1097/COH.0000000000000270.

\title{
Bone Health in HIV-infected Children and Adolescents
}

\author{
Allison Ross Eckard ${ }^{1}$ and Stefano Mora ${ }^{2}$ \\ ${ }^{1}$ Medical University of South Carolina, Charleston, South Carolina, USA \\ ${ }^{2}$ Laboratory of Pediatric Endocrinology and Pediatric Bone Densitometry Service, Division of \\ Genetics and Cell Biology, IRCCS San Raffaele Scientific Institute, Milano, Italy
}

\begin{abstract}
Purpose of review-Chronic HIV infection and exposure to antiretroviral therapy compromises bone health in children and adolescents, potentially impacting their long-term quality of life. Thus, the purpose of this paper is to review the most recent literature on this topic in HIV-infected children and adolescents.
\end{abstract}

Recent findings-Recent studies continue to demonstrate bone abnormalities in HIV-infected children and adolescents, whether HIV is acquired perinatally or during adolescence. Researchers have employed new modalities, both high tech and those that can be utilized in resource-limited settings, to better assess bone health. New data suggest that this population may also be experiencing an increase incidence of factures, and they may not acquire the same peak bone mass as their HIV-uninfected counterparts. Reassuringly, however, in utero tenofovir exposure does not appear to have a significant impact on bone health in HIV-exposed, uninfected infants.

Summary-Recent findings underscore the need for further research on bone in HIV-infected children and adolescents. Longitudinal studies are especially needed to evaluate long-term risk of osteoporosis and fractures, since this population is exposed to HIV and antiretroviral therapy for many decades starting early in life and during the most critical time for skeletal growth and bone mass accrual.

\section{Keywords}

HIV; pediatrics; bone mineral density; vitamin D; HIV-exposed infants

\section{Introduction}

HIV-infected adults exhibit an increased risk of osteoporosis and factures. Bone health appears to be compromised in HIV-infected children and adolescents too, although the longterm ramifications are largely unknown. Bone toxicity is particularly concerning in this younger population, however, as bone mass acquisition occurs during this period of rapid

Correspondence and Reprints: Allison Ross Eckard, MD, Associate Professor of Pediatrics and Medicine, Divisions of Infectious Diseases, Medical University of South Carolina, 135 Rutledge Ave, Ste 1217, MSC 752 Charleston, SC 29425; Phone: 843-792-9909; Fax: 843-792-5127; eckarda@ musc.edu.

Conflicts of interest

ARE has received research funding from Bristol-Myers Squibb, Cubist Pharmaceuticals, and GlaxoSmithKline and has served as an advisor and speaker for Gilead. SM has no conflicts of interest. 
growth, and the effects of chronic HIV infection and exposure to antiretroviral therapy (ART) accumulate over many decades (Figure 1). This review summarizes the most recent data on bone health in HIV-infected children and adolescents.

\section{Assessing Bone Health in HIV-Infected Children and Adolescents}

\section{Fracture Risk}

Data on fracture risk in the pediatric population are scarce. A multicenter, prospective cohort study from 2004-2007 did not show an increased incidence of fractures in HIV-infected children compared to HIV-exposed, uninfected (HEU) children ${ }^{1}$. These results were then compared to the incidence of fractures in a similar subsequent study conducted from 2008$2014^{2}$. The authors observed higher fracture rates in the second study compared to the first, even after adjustment for age, suggesting that fracture rates may be increasing in HIVinfected children and adolescents. Further studies are needed, though, to confirm these findings.

\section{Bone Mineral Density by Dual-Energy X-Ray Absorptiometry}

While there are few studies evaluating fracture risk in HIV-infected children and adolescents, multiple cross-sectional studies have evaluated bone mineral density (BMD) and bone mineral content (BMC) in this population. Usually measured by dual-energy x-ray absorptiometry (DXA), decreased BMD and BMC have been demonstrated across ages, sex, sexual maturity stages, and HIV acquisition modes ${ }^{3-6}$. One concern has been whether they exhibit increases in bone mass and maintain or improve their BMD z-scores over time. This is particularly important during puberty because $\sim 80 \%$ of the adult bone mass accrues during this time. Low peak bone mass is related to an increased risk of osteoporosis and fractures later in life ${ }^{7}$.

A prospective cohort study of 35 pre-pubertal HIV-infected children in Brazil were evaluated for bone mass accrual by measuring DXA at baseline and again $24 \pm 6$ months later ${ }^{6}$. At the second time point, subjects were divided into pre-pubertal and pubertal groups. Although both groups showed an increase in bone mass, pubertal subjects expectedly showed more of an increase for BMC, total body (TB) BMD, and lumbar spine (LS) BMD compared to prepubertal subjects. Of concern, however, was that TB BMD z-scores actually worsened over time for the entire cohort, while LS stayed the same. Additionally, pre-pubertal subjects had worse TB and LS BMD z-scores at the second time point compared to the pubertal subjects. In fact, while only $5.7 \%$ of subjects had LS z-scores $\leq 2.5$ at baseline, at the second time point, this percentage increased to $31.3 \%$ among pre-pubertal subjects compared to $5.3 \%$ for pubertal subjects. This may be because height-for-age and BMI z-score decreased over time and were lower in the pre-pubertal group, possible signs of delayed puberty, which is common in perinatal HIV infection ${ }^{8}$. In addition, changes in body composition can affect the accuracy of serial DXA measurements, which may have impacted the results of this study ${ }^{9}$.

\section{Quantitative Computed Tomography}

Based on the results of the aforementioned study, one could argue that measuring BMD after sexual maturity would be a better indicator of long-term bone health. This was the approach 
taken in another recent study which used high-resolution peripheral quantitative computed tomography (HR-pQCT) to assess bone health in HIV-infected young adults after peak bone mass had likely been achieved ${ }^{10}$.

Although DXA is most commonly used to assess BMD, it has limitations. Dual-energy x-ray absorptiometry calculates BMD by providing a two-dimensional measurement of a threedimensional structure derived by dividing BMC by the apparent area of the analyzed bone, often referred to as areal BMD (aBMD). Consequently, DXA overestimates BMD in taller people and underestimates BMD in smaller people. Because HIV-infected children and adolescents often have impaired growth and delayed onset of puberty, DXA may underestimate BMD in this population.

Therefore, Yin, et al, used HR-pQCT to measure true volumetric BMD (vBMD) and compare the results to aBMD obtained by DXA in $30 \mathrm{HIV}$-infected African-American or Hispanic Tanner stage 5 men aged 20-25 on ART and 15 HIV-uninfected controls. They found that the HIV-infected men had lower aBMD and aBMD z-scores by DXA at all sites measured, whether infected perinatally or during adolescence. Importantly, the decrease in aBMD was not due to smaller bone size, as cross-sectional area was similar by DXA and HR-pQCT at the radius and tibia. Moreover, total vBMD was significantly lower at both radius and tibia as assessed by HR-pQCT.

Another advantage of HR-pQCT is that it can differentiate trabecular from cortical bone and assess bone microarchitecture. Here, the authors found that trabecular microarchitecture was markedly abnormal at the tibia and radius in HIV-infected men (regardless of mode of HIV acquisition), showing thinner, more widely separated and heterogeneously distributed trabeculae. Similarly, HIV-infected men had lower cortical area, significantly thinner cortices, and lower estimated whole bone and trabecular stiffness. These data suggest that HIV infection acquired early in life (either perinatally or during adolescence) is associated with markedly abnormal bone microarchitecture, lower peak bone mass, and decreased bone strength, which may place them at a higher risk of osteoporosis and fractures later in life.

\section{Quantitative Ultrasound}

Arguably, using HR-pQCT to measure vBMD and bone microarchitecture is an exceptionally precise and comprehensive method for assessing bone health; however, it is an expensive and impractical technique in many settings. Quantitative ultrasonography (QUS) assesses bone quality by measuring the attenuation and speed of an ultrasound wave through bone. Unlike HR-pQCT and DXA, QUS does not require high-level training or radiation exposure, is portable, and has a short scan time, making it potentially advantageous for assessing and following bone health.

Arpadi, et al, evaluated the inter-rater reliability of QUS and the relationship between QUS and DXA in a cohort of South African HIV-infected children ${ }^{11}$. In 47 perinatally-infected children on ART, the authors measured speed of sound (SoS) and broadband ultrasound attenuation (BUA) at the heel/calcaneus with QUS and calculated a calcaneus stiffness index (SI) utilizing both measurements. They also measured trans-axial SoS along the distal 1/3 radius with a hand-held ultrasound probe. Both broadband ultrasound attenuation and SI of 
the calcaneus moderately correlated with whole-body and LS BMC and BMD measured by DXA, but SOS by itself varied between operators and did not correlate well with DXA measurements. Using the hand-held ultrasound, on the other hand, SoS measurements of the $1 / 3$ radius were highly reliable between the two operators and correlated significantly with DXA measurements. Another recent study showed that QUS was an independent predictor of fracture in adult men and women, albeit a weaker predictor compared to DXA ${ }^{12}$. Thus, although additional information is needed, these data suggest that QUS may be valuable in assessing bone health in HIV-infected children in resource-limited settings.

\section{Anthropometric Predictive Models}

In situations where even ultrasound is impractical, anthropometry may offer an alternative for bone health assessment. With this idea in mind, Lima, et al, developed predictive equations for whole-body BMC and aBMD based on anthropometric measures in perinatally-infected children and adolescents in Brazil using DXA as the reference method. Using height, weight, arm circumference, body mass index, and femoral diameter, with age and skin color adjusted by sex, the models predicted $84 \%$ and $94 \%$ of aBMD and BMC, respectively. With subsequent validation in other populations, these models could allow an alternative for bone health monitoring in resource-limited settings ${ }^{13}$.

\section{Bone Metabolism Alterations}

HIV infection affects BMD and bone microarchitecture, but the mechanisms are poorly understood. There is evidence that the rate of bone metabolism is altered in HIV-infected youth, in whom an accelerated bone resorption and an increased bone formation have been described ${ }^{14}$. Rates of bone metabolism can be assessed by measurements of specific markers of bone formation and bone resorption. In children and adolescents, the serum concentration of the specific markers mimic the growth rate, with higher levels during infancy and adolescence ${ }^{15,16}$. The regulation of osteoblast differentiation and activity is maintained by the Wnt/ $\beta$-catenin pathway ${ }^{17}$, which is fine-tuned by a large number of extracellular proteins. Sclerostin and Dkk1, among others, are potent inhibitors, and their concentrations are measurable in serum ${ }^{17}$.

A recent study was aimed at unraveling the regulatory mechanism responsible for the alterations in bone formation in HIV-infected youth ${ }^{18}$. The serum concentrations of bonespecific alkaline phosphatase (BAP), sclerostin, and Dkk1 were measured in a group of 54 HIV-infected children and adolescents and 105 healthy controls. HIV-infected subjects had significantly higher BAP concentrations compared to the healthy peers. This higher bone formation rate was coupled with significantly lower serum concentrations of both sclerostin and Dkk1. The patients were also grouped according to the type of antiretroviral (ARV) they were receiving, but no differences were observed between the groups. Although an influence of ARVs on bone metabolism could not be excluded due to the small number of observations in each group, the increased bone formation observed in HIV-infected young patients appeared to be related to an imbalanced control of osteoblast activity due to low concentrations of inhibitors of the $\mathrm{Wnt} / \beta$-catenin pathway. 


\section{Role of Vitamin D Deficiency}

Vitamin D is essential for optimal bone health. There is evidence that many HIV-infected patients have vitamin D insufficiency, as measured by blood levels of 25 -hydroxyvitamin D $(25(\mathrm{OH}) \mathrm{D})^{19-22}$, but data regarding vitamin D deficiency and bone health in the pediatric population are scarce. A recent study in perinatally-infected HIV-infected children and adolescents showed that BMD measurements tended to be lower in subjects with a lower serum 25(OH)D concentration ${ }^{23}$.

Few randomized, placebo-controlled trials of vitamin D supplementation have been conducted in HIV-infected children and adolescents ${ }^{24-28}$, and only two studies to date have evaluated bone changes with supplementation ${ }^{24,27}$. In Eckard, et al, authors investigated changes in BMD after 12 months of supplementation in 102 HIV-infected youth 8-26 years old on ART with baseline 25(OH)D $330 \mathrm{ng} / \mathrm{mL}^{27}$. In this randomized, active-control, double-blind trial, HIV-infected subjects receiving either 60,000 or $120,000 \mathrm{IU} / \mathrm{month}$ had significant increases in BMD at the LS and hip over the 12 months. In subjects receiving only $18,000 \mathrm{IU} /$ month, increases were seen in BMD for hip, but not LS. Bone turnover markers also decreased significantly in subjects receiving the higher doses.

Another recent study performed on a cohort of 58 HIV-infected youths assessed the effect of a daily high-dose supplementation with 7,000 IU of vitamin $\mathrm{D}^{28}$. An interesting finding was related to the unchanged parathyroid hormone (PTH) concentration observed despite the increase in $25(\mathrm{OH}) \mathrm{D}$. The majority of subjects were vitamin D deficient at baseline, so one would have expected a decrease in PTH with vitamin D supplementation which was not the case. The hypothesis was that there may be a direct effect of ARVs on PTH secretion, but further studies are necessary.

\section{Role of Tenofovir Disoproxil Fumarate}

Tenofovir disoproxil fumarate (TDF) is associated with greater bone loss than other ARVs; however, its use is widespread because of its excellent efficacy for maintaining virological suppression. Recently, Aurpibul, et a ${ }^{29}$, conducted a prospective, open-label study in HIVinfected children where 40 were started on a TDF-containing regimen, and 40 age-, genderand CD4-matched controls received a TDF-sparing regimen. Spine BMD z-scores decreased significantly over 96 weeks in children receiving TDF, but were unchanged in the TDFsparing group. Most of this decrease occurred over the first 24 weeks of the study, then remained stable thereafter. At week 96, there was no significant difference in the prevalence of LS BMD z-scores $<-2.0$ between the two groups.

The final results of the Gilead 321 extension study were also recently published, which included HIV-infected subjects ages 12-17 years receiving TDF for a median duration of 96 weeks ${ }^{30}$. Results varied depending on the body part and what BMD measurement was reported. Although both LS and TB less head (TBLH) BMD increased over time, ageadjusted LS and TBLH BMD z-scores declined significantly over 96 and 192 weeks, respectively. However, height-age-adjusted LS BMD z-scores were unchanged from baseline to week 96 and showed significant increases from week 144. Moreover, although height- 
age-adjusted TB BMD Z-scores decreased from baseline to week 48, median changes after this were not significantly different from baseline.

Similarly, long-term safety data from Gilead 352 were recently presented ${ }^{31}$, which included children aged 2-15 years in a phase 3, randomized, open-label non-inferiority study comparing switching to TDF vs. continuing on stavudine or zidovudine ${ }^{32}$. Bone data presented were limited, but TB, TBLH, and LS height-age-adjusted BMD z-scores stayed the same or improved slightly over 336 weeks. Results from both Gilead studies, though, must be interpreted cautiously, as data are limited by a lack of comparator and small sample sizes. Longer-term studies are needed to fully assess the clinical impact of TDF on bone. However, tenofovir alafenamide (TAF), Gilead's second generation tenofovir pro-drug, may replace TDF over time. Thus far, data have only been presented in a small number of HIVinfected ART-naïve adolescents, but showed minimal change in height-adjusted BMD zscores over 24 weeks $^{33}$.

\section{Bone Health in HIV-Exposed Infants}

Although there are concerns about fetal growth and bone toxicity from animal studies 34,35 , TDF use during pregnancy has increased in recent years. However, the effect of prenatal TDF exposure on growth and bone health is still debated. Previous studies have suggested a potential side effect of maternal TDF use on infant growth ${ }^{36,37}$, but others have not ${ }^{38,39}$.

Recently, studies have focused more on the assessment of bone health in HIV-exposed uninfected (HEU) infants. A large study compared BMC in 69 TDF-exposed infants to that of 74 TDF-unexposed infants ${ }^{40}$. Bone mineral content was measured by DXA in the whole skeleton at a mean age of 4-5 weeks. The unadjusted comparison showed a 7.8g difference between exposure groups, with higher BMC in the TDF-unexposed infants. After adjustment for maternal age at delivery, tobacco use during pregnancy, infant race, gestational age, length, age at DXA scan, and clinical site, the difference between exposure groups decreased to 5.5g. Interestingly, when the analysis was repeated considering BMC less head, the difference between the two groups was no longer present.

In another study, bone metabolism rate was assessed in 103 infants of Malawian origin, born from mothers treated with TDF during pregnancy ${ }^{41}$. Growth measurements were collected at 6 and 12 months of age, and BAP and C-terminal telopeptide of type I collagen were measured in serum. The study did not show growth impairment, and there were no association between growth indexes and ART duration during pregnancy. Similarly, bone metabolism indexes were not altered in HEU infants.

Finally, an interesting approach to monitor the degree of TDF exposure was recently proposed $^{42}$. The study was designed to verify whether TDF quantified in the meconium was associated with growth and bone outcomes among HEU infants. Whole-body BMC was measured at 2 weeks of age in 58 infants. Growth measurements were not associated with meconium TDF concentration. Similarly, meconium TDF concentration was not correlated with BMC in univariate or multivariate models. The results of this study support the 
observations of previous studies that failed to show associations between maternal TDF treatment duration and infant growth or bone outcomes ${ }^{36-38}$.

\section{Conclusions}

While ART has allowed HIV-infected children and adolescents to live decades longer than before, they have evidence of compromised bone health even at their young age. New studies suggest that they may be at a high risk of osteoporosis and fractures later in life, although clinical manifestations during youth are rare. Optimizing bone health in HIVinfected youth is crucial to minimize the risk of long-term bone complications. These include ensuring sufficient calcium and vitamin D intake, encouraging weight-bearing exercise, and minimizing modifiable risk factors, such as smoking ${ }^{43}$. It is also the opinion of the authors to periodically measure $25(\mathrm{OH})$ D concentrations and supplement as necessary. Although routine monitoring via DXA cannot be recommended at this time, baseline and periodic DXA should be considered in high-risk patients. Long-term, continued research on assessing bone health and novel interventions to minimize bone loss are urgently needed in this population. Reassuringly, new data suggest that HEU infants exposed to TDF in utero may not experience the same bone toxicity as their HIV-infected counterparts.

\section{Acknowledgments}

None.

Financial support and sponsorship

This work was made possible in part by the National Institute of Child Health and Development at the National Institutes of Health [K23 HD069199 to ARE]. The content is solely the responsibility of the authors and does not necessarily represent the official views of the National Institutes of Health.

\section{References}

1. Siberry GK, Li H, Jacobson D. Pediatric ACTGCS. Fracture risk by HIV infection status in perinatally HIV-exposed children. AIDS research and human retroviruses. Mar; 2012 28(3):247250. [PubMed: 22471877]

2•. Mirani G, Williams PL, Chernoff M, et al. Changing Trends in Complications and Mortality Rates Among US Youth and Young Adults With HIV Infection in the Era of Combination Antiretroviral Therapy. Clinical infectious diseases : an official publication of the Infectious Diseases Society of America. Dec 15; 2015 61(12):1850-1861. This study compares the incidence of complications and deaths in two US-based prospective multicenter cohort study conducted from 2004-2007 and from 2008-2014. The data suggest that fracture rates may be increasing in HIV-infected children and adolescents. [PubMed: 26270680]

3. DiMeglio LA, Wang J, Siberry GK, et al. Bone mineral density in children and adolescents with perinatal HIV infection. Aids. Jan 14; 2013 27(2):211-220. [PubMed: 23032412]

4. Jacobson DL, Lindsey JC, Gordon CM, et al. Total body and spinal bone mineral density across Tanner stage in perinatally HIV-infected and uninfected children and youth in PACTG 1045. Aids. Mar 13; 2010 24(5):687-696. [PubMed: 20168204]

5. Mulligan K, Harris DR, Emmanuel P, et al. Low bone mass in behaviorally HIV-infected young men on antiretroviral therapy: Adolescent Trials Network Study 021B. Clinical infectious diseases : an official publication of the Infectious Diseases Society of America. Aug; 2012 55(3):461-468.

[PubMed: 22573848]

6•. Palchetti CZ, Szejnfeld VL, de Menezes Succi RC, et al. Impaired bone mineral accrual in prepubertal HIV-infected children: a cohort study. The Brazilian journal of infectious diseases : 
an official publication of the Brazilian Society of Infectious Diseases. Nov-Dec;2015 19(6):623630. This study compared bone mass accrual over a 24-month period in subjects who remained pre-pubertal vs. those who began puberty during the study period. Alarmingly, pre-pubertal subjects had worse total body and lumbar spine BMD z-scores at study conclusion compared to the pubertal subjects.

7. Heaney RP, Abrams S, Dawson-Hughes B, et al. Peak bone mass. Osteoporosis international : a journal established as result of cooperation between the European Foundation for Osteoporosis and the National Osteoporosis Foundation of the USA. 2000; 11(12):985-1009.

8. Buchacz K, Rogol AD, Lindsey JC, et al. Delayed onset of pubertal development in children and adolescents with perinatally acquired HIV infection. Journal of acquired immune deficiency syndromes. May 1; 2003 33(1):56-65. [PubMed: 12792356]

9. Yu EW, Thomas BJ, Brown JK, Finkelstein JS. Simulated increases in body fat and errors in bone mineral density measurements by DXA and QCT. Journal of bone and mineral research : the official journal of the American Society for Bone and Mineral Research. Jan; 2012 27(1):119-124.

10. Yin MT, Lund E, Shah J, et al. Lower peak bone mass and abnormal trabecular and cortical microarchitecture in young men infected with HIV early in life. Aids. Jan 28; 2014 28(3):345-353. [PubMed: 24072196]

11. Arpadi, SM.; Shiau, S.; Kuhn, L., et al. Bone quality determination by ultrasonometry in young South African HIV-infected children. Paper presented at: 22nd Conference on Retroviruses and Opportunistic Infections; 2015; Seattle, Washington. Abstract 920

12. McCloskey EV, Kanis JA, Oden A, et al. Predictive ability of heel quantitative ultrasound for incident fractures: an individual-level meta-analysis. Osteoporosis international : a journal established as result of cooperation between the European Foundation for Osteoporosis and the National Osteoporosis Foundation of the USA. Jul; 2015 26(7):1979-1987.

13. Lima LR, Krug RR, Silva RC, et al. Prediction of Areal Bone Mineral Density and Bone Mineral Content in Children and Adolescents Living With HIV Based on Anthropometric Variables. Journal of clinical densitometry : the official journal of the International Society for Clinical Densitometry. Jul 20.2015

14. Vigano A, Zuccotti GV, Puzzovio M, et al. Tenofovir disoproxil fumarate and bone mineral density: a 60-month longitudinal study in a cohort of HIV-infected youths. Antiviral therapy. 2010; 15(7):1053-1058. [PubMed: 21041922]

15. Rauchenzauner M, Schmid A, Heinz-Erian P, et al. Sex- and age-specific reference curves for serum markers of bone turnover in healthy children from 2 months to 18 years. The Journal of clinical endocrinology and metabolism. Feb; 2007 92(2):443-449. [PubMed: 17105843]

16•. Mora S, Cafarelli L, Erba P, et al. Differential effect of age, gender and puberty on bone formation rate assessed by measurement of bone-specific alkaline phosphatase in healthy Italian children and adolescents. Journal of bone and mineral metabolism. 2009; 27(6):721-726. Mora, et al, shed some light onto the mechanisms by which compromised bone health occurs in HIV-infected children and adolescents by observing higher serum concentrations of BAP, a bone formation marker, coupled with lower serum concentrations of sclerostin and Dkk1, bone formation inhibitors. [PubMed: 19455384]

17. Krishnan V, Bryant HU, Macdougald OA. Regulation of bone mass by Wnt signaling. The Journal of clinical investigation. May; 2006 116(5):1202-1209. [PubMed: 16670761]

18. Mora S, Puzzovio M, Giacomet V, et al. Sclerostin and DKK-1: two important regulators of bone metabolism in HIV-infected youths. Endocrine. Aug; 2015 49(3):783-790. [PubMed: 25596857]

19. Viard JP, Souberbielle JC, Kirk O, et al. Vitamin D and clinical disease progression in HIV infection: results from the EuroSIDA study. Aids. Jun 19; 2011 25(10):1305-1315. [PubMed: 21522006]

20. Vescini F, Cozzi-Lepri A, Borderi M, et al. Prevalence of hypovitaminosis D and factors associated with vitamin D deficiency and morbidity among HIV-infected patients enrolled in a large Italian cohort. Journal of acquired immune deficiency syndromes. Oct 1; 2011 58(2):163-172. [PubMed: 21826011]

21. Eckard AR, Tangpricha V, Seydafkan S, et al. The relationship between vitamin D status and HIVrelated complications in HIV-infected children and young adults. The Pediatric infectious disease journal. Nov; 2013 32(11):1224-1229. [PubMed: 23360833] 
22. Eckard AR, Judd SE, Ziegler TR, et al. Risk factors for vitamin D deficiency and relationship with cardiac biomarkers, inflammation and immune restoration in HIV-infected youth. Antiviral therapy. 2012; 17(6):1069-1078. [PubMed: 22894927]

23. Jacobson, DL.; Mitchell, G.; Stephensen, CB., et al. Vitamin D status and bone outcomes in perinatally HIV-infected children. Paper presented at: 22nd Conference on Retroviruses and Opportunistic Infections; 2015; Seattle, Washington. Abstract 931

24. Arpadi SM, McMahon D, Abrams EJ, et al. Effect of bimonthly supplementation with oral cholecalciferol on serum 25-hydroxyvitamin D concentrations in HIV-infected children and adolescents. Pediatrics. Jan; 2009 123(1):e121-126. [PubMed: 19117833]

25. Havens PL, Mulligan K, Hazra R, et al. Serum 25-hydroxyvitamin D response to vitamin D3 supplementation 50,000 IU monthly in youth with HIV-1 infection. The Journal of clinical endocrinology and metabolism. Nov; 2012 97(11):4004-4013. [PubMed: 22933542]

26. Giacomet V, Vigano A, Manfredini V, et al. Cholecalciferol supplementation in HIV-infected youth with vitamin D insufficiency: effects on vitamin D status and T-cell phenotype: a randomized controlled trial. HIV clinical trials. Mar-Apr;2013 14(2):51-60. [PubMed: 23611825]

27. Eckard, AR.; Chahroudi, A.; Rosebush, JC., et al. Effects of vitamin D supplementation on BMD and bone markers in HIV+ youth. Paper presented at: 23rd Conference on Retroviruses and Opportunistic Infections; 2016; Boston, MA.

28. Stallings VA, Schall JI, Hediger ML, et al. High-dose vitamin D3 supplementation in children and young adults with HIV: a randomized, placebo-controlled trial. The Pediatric infectious disease journal. Feb; 2015 34(2):e32-40. [PubMed: 24988118]

29•. Aurpibul L, Cressey TR, Sricharoenchai S, et al. Efficacy, safety and pharmacokinetics of tenofovir disoproxil fumarate in virologic-suppressed HIV-infected children using weight-band dosing. The Pediatric infectious disease journal. Apr; 2015 34(4):392-397. Subjects started on TDF showed significant decreases in LS BMD z-scores over the 96 week-study period, mostly during the first 24 weeks. In comparison, subjects on TDF-sparing regimens did not have any significant decreases in BMD z-scores over the study period. [PubMed: 25760566]

30. Della Negra M, De Carvalho AP, De Aquino MZ, et al. Long-term efficacy and safety of tenofovir disoproxil fumarate in HIV-1-infected adolescents failing antiretroviral therapy: the final results of study GS-US-104-0321. The Pediatric infectious disease journal. Apr; 2015 34(4):398-405. [PubMed: 25599284]

31. Saez-Llorens, X.; Deville, JG.; Mirza, A., et al. Long-term safety and efficacy of tenofovir DF therapy in HIV-infected children through week 336. Paper presented at: 22nd Conference on Retroviruses and Opportunistic Infections; 2015; Seatle, Washington. Abstract 954

32. Saez-Llorens X, Castano E, Rathore M, et al. A randomized, open-label study of the safety and efficacy of switching stavudine or zidovudine to tenofovir disoproxil fumarate in HIV-1-infected children with virologic suppression. The Pediatric infectious disease journal. Apr; 2015 34(4): 376-382. [PubMed: 25760565]

33. Kizito, H.; Gaur, A.; Prasitsuebsai, W., et al. Safety, efficacy and pharmacokinetics of the integrase inhibitor-based E/C/F/TAF single-tablet regimen in treatment-naïve HIV-infected adolescents through 24 weeks of treatment. Paper presented at: 22nd Conference on Retroviruses and Opportunistic Infections; 2015; Seattle, Washington. Abstract 953

34. Tarantal AF, Castillo A, Ekert JE, Bischofberger N, Martin RB. Fetal and maternal outcome after administration of tenofovir to gravid rhesus monkeys (Macaca mulatta). Journal of acquired immune deficiency syndromes. Mar 1; 2002 29(3):207-220. [PubMed: 11873070]

35. Van Rompay KK, Durand-Gasselin L, Brignolo LL, et al. Chronic administration of tenofovir to rhesus macaques from infancy through adulthood and pregnancy: summary of pharmacokinetics and biological and virological effects. Antimicrobial agents and chemotherapy. Sep; 2008 52(9): 3144-3160. [PubMed: 18573931]

36. Siberry GK, Williams PL, Mendez H, et al. Safety of tenofovir use during pregnancy: early growth outcomes in HIV-exposed uninfected infants. Aids. Jun 1; 2012 26(9):1151-1159. [PubMed: 22382151]

37. Ransom CE, Huo Y, Patel K, et al. Infant growth outcomes after maternal tenofovir disoproxil fumarate use during pregnancy. Journal of acquired immune deficiency syndromes. Dec 1; 2013 64(4):374-381. [PubMed: 24169122] 
38. Vigano A, Mora S, Giacomet V, et al. In utero exposure to tenofovir disoproxil fumarate does not impair growth and bone health in HIV-uninfected children born to HIV-infected mothers. Antiviral therapy. 2011; 16(8):1259-1266. [PubMed: 22155907]

39. Gibb DM, Kizito H, Russell EC, et al. Pregnancy and infant outcomes among HIV-infected women taking long-term ART with and without tenofovir in the DART trial. PLoS medicine. 2012; 9(5):e1001217. [PubMed: 22615543]

40. Siberry GK, Jacobson DL, Kalkwarf HJ, et al. Lower Newborn Bone Mineral Content Associated With Maternal Use of Tenofovir Disoproxil Fumarate During Pregnancy. Clinical infectious diseases : an official publication of the Infectious Diseases Society of America. Sep 15; 2015 61(6):996-1003. [PubMed: 26060285]

41. Liotta, G.; Floridia, M.; Andreotti, M., et al. Growth and bone markers in Malawian infants preand postnatally exposed to tenofovir. Paper presented at: 22nd Conference on Retroviruses and Opportunistic Infections; 2015; Seattle, Washington. Abstract 879

42• Himes SK, Wu JW, Jacobson DL, et al. Meconium Tenofovir Concentrations and Growth and Bone Outcomes in Prenatally Tenofovir Exposed HIV-Uninfected Children. The Pediatric infectious disease journal. Aug; 2015 34(8):851-857. This study quantified meconium TDF concentrations to determine if they were associated with growth and bone outcomes in HEU infants. Growth parameters and BMC were not associated with meconium TDF concentrations, which is reassuring given the increased use of TDF during pregnancy. [PubMed: 25961889]

43. Panel on Antiretroviral Therapy and Medical Management of HIV-Infected Children. [Accessed on February 1, 2016] Guidelines for the Use of Antiretroviral Agents in Pediatric HIV Infection. Available at http://aidsinfo.nih.gov/contentfiles/lvguidelines/pediatricguidelines.pdfPage K-37, Table 13j 


\section{Key Points}

- HIV-infected children and adolescents, whether infected perinatally or later in adolescence, have compromised bone health compared to their healthy uninfected counterparts.

- The bone changes observed in HIV-infected children and adolescents include alterations in the rate of bone metabolism, decreases in BMD, and markedly abnormal bone microarchitecture.

- $\quad$ Although DXA is the most commonly used modality to assess BMD in HIVinfected children and adolescents, HR-pQCT offers a more comprehensive and precise method for assessing bone health, while QUS and prediction models based on anthropometrics may offer inexpensive alternatives in resource-limited settings.

- The compromised bone health in HIV-infected children and adolescents is multifactorial, including traditional risk factors like vitamin D deficiency, as well as direct and indirect effects of HIV infection and ART, especially TDF.

- Studies to date have failed to show that maternal TDF treatment is associated with appreciable changes in growth or bone outcomes in HIV-exposed, but uninfected infants. 

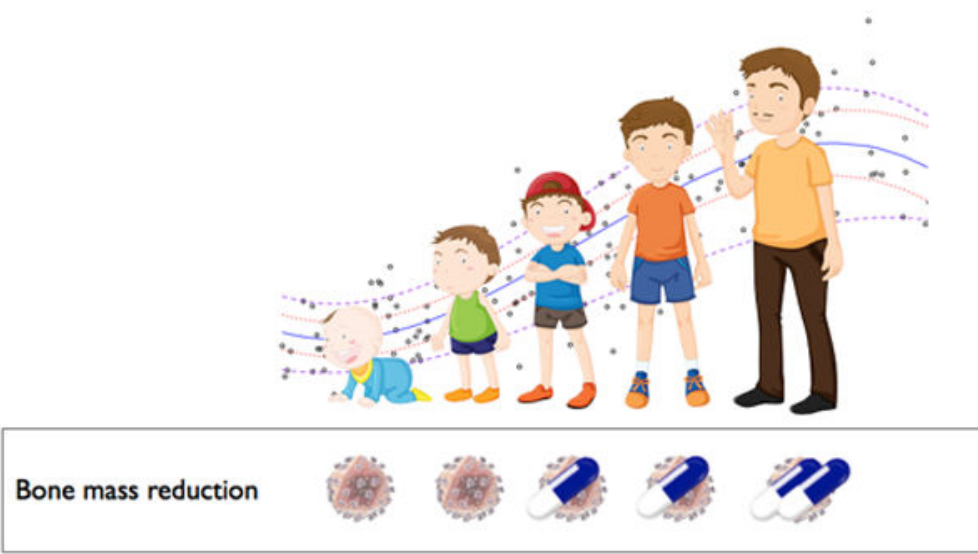

Bone metabolism alterations
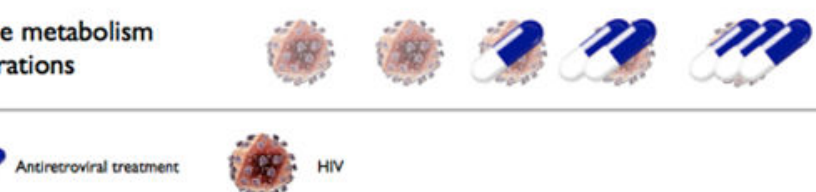

Figure 1. Timing of the effect of antiretroviral therapy on bone mass and bone metabolism The available data indicate that the skeletal system of infants is not affected. The negative outcome begins in childhood and worsens with age with the most significant effects during puberty when the majority of bone mass is accumulated. The role of HIV is still unclear, although in vitro studies show a direct effect of the virus on bone cells. 